\title{
Contributions to the Galactic halo from in-situ, kicked-out, and accreted stars
}

\author{
Allyson A. Sheffield ${ }^{1}$, Kathryn V. Johnston ${ }^{2}$, Katia Cunha ${ }^{3}$, \\ Verne V. Smith ${ }^{4}$ and Steven R. Majewski ${ }^{5}$ \\ ${ }^{1}$ LaGuardia Community College, City University of New York, \\ Department of Natural Sciences, 31-10 Thomson Ave., Long Island City, NY, 11101, USA \\ email: asheffield@lagcc.cuny.edu \\ ${ }^{2}$ Columbia University, Dept. of Astronomy, Mail Code 5246, New York, NY, 10027, USA \\ ${ }^{3}$ Observatório Nacional, Rua General José Cristino, 77, 20921-400 São Cristóvão, Rio de \\ Janeiro, RJ, Brazil \\ ${ }^{4}$ National Optical Astronomy Observatories, Tucson, AZ, 98765, USA \\ ${ }^{5}$ University of Virginia, Dept. of Astronomy, P.O. Box 400325, Charlottesville, VA, 22904, USA
}

\begin{abstract}
We report chemical abundances for a sample of $66 \mathrm{M}$ giants with high $\mathrm{S} / \mathrm{N}$ highresolution spectroscopy in the inner halo of the Milky Way. The program giant stars have radial velocities that vary significantly from those expected for stars moving on uniform circular orbits in the Galactic disk. Thus, based on kinematics, we expect a sample dominated by halo stars. Abundances are derived for $\alpha$-elements and neutron capture elements. By analyzing the multidimensional abundance space, the formation site of the halo giants - in-situ or accreted - can be assessed. Of particular interest are a class of stars that form in-situ, deep in the Milky Way's gravitational potential well, but are "kicked out" of the disk into the halo due to a perturbation event. We find: (1) our sample is dominated by accreted stars and (2) tentative evidence of a small kicked-out population in our Milky Way halo sample.
\end{abstract}

Keywords. Galaxy: halo, Galaxy: structure, stars: abundances

\section{Introduction}

Where did stars in the Milky Way's halo form? The $\Lambda$ CDM model predicts that the Milky Way's halo was built in a "bottom-up" fashion, and this view is now generally accepted due to overwhelming evidence of the relics of past mergers. It is still uncertain, however, what fraction of the halo is made up of such accreted debris. Close to the time of accretion, a group of stars formed in a particular satellite of the Milky Way will show coherence spatially, kinematically, and chemically. In the inner halo where dynamical timescales are short, spatial coherence will become blurred quickly, although kinematical and chemical coherence remain. Kinematics alone may still lead to ambiguity, as a merger event can cause stars formed in the Milky Way to redistribute into rings in the halo and these rings can be difficult to distinguish from accreted satellite stars. Thus, to get a more complete profile of a star's formation history, both kinematical and chemical information are needed.

Stars form in gas clouds, and the chemical patterns of stars reflect successive generations of stellar evolution within the cloud. Due to their formation in Type II supernovae, $\alpha$-elements probe the relative timescale of formation for populations of stars and are sensitive to the star formation efficiency of the progenitor. Thus, a star that formed in a dSph progenitor will occupy a different region in the $[\alpha / \mathrm{Fe}]-[\mathrm{Fe} / \mathrm{H}]$ plane than a star formed in a Milky Way-sized progenitor. The addition of $r-$ and s-process elements gives a more complete evolutionary picture of the star's formation site. The yields of $r$ - and 
s-process elements, which are synthesized in Type II supernovae and thermally pulsating AGB stars, respectively, are coupled to the Fe seed nuclei present in the formation site; thus, the yields of neutron capture elements vary with metallicity and provide further constraints on a star's formation scenario.

As detailed in Sheffield et al. (2012), three possible formation scenarios for halo stars can be identified: in-situ halo stars, which formed in the Milky Way's main dark matter (DM) halo; kicked-out disk stars, which formed much deeper within the potential well of the Milky Way's dominant DM halo and were subsequently ejected into the halo due to a perturbation event; accreted stars, which formed in a separate DM subhalo. Kickedout disk stars will have chemical abundance patterns similar to the Milky Way's disk but halo-like kinematics. We note that an additional class is also possible, wherein stars that originated in a fairly massive satellite were accreted and dragged into the plane of the disk, thus forming a population of "accreted disk stars" with disk-like kinematics but chemistry reflective of the satellite's DM subhalo (Read et al. 2008).

In this study, we use M giants selected from 2MASS to assess the formation scenario of stars in the inner halo. M giants are a useful probe of the formation history of the nearby halo: they are old, metal-rich stars and thus are likely to be found in the thick disk and the metal-rich tail of the halo. They are also sensitive to more recent accretion (Sharma et al. 2011) so surveys isolating M giants will tend to pick up stars associated with luminous, metal-rich satellites. This was seen in the work of Majewski et al. 2003, in which they mapped the Galaxy in M giants and the Sgr core and northern and southern streams were strikingly revealed. We present an expanded study of Sheffield et al. (2012), with nearly double the sample size of $\mathrm{M}$ giants and the addition of neutron capture elements.

\section{Methodology}

\subsection{Chemical Tagging}

In this contribution, we present results for $69 \mathrm{M}$ giants with high $\mathrm{S} / \mathrm{N}$, high-resolution spectroscopy, 3 of which are $\mathrm{M}$ giants selected as thick disk calibration stars. The stars were selected from a medium-resolution survey of $1799 \mathrm{M}$ giants (Sheffield et al. 2012), and the selection criteria for medium-resolution spectroscopy and high-resolution followup is detailed in that work. Sheffield et al. (2012) presented the results for stars in the $[\mathrm{Ti} / \mathrm{Fe}]-[\mathrm{Fe} / \mathrm{H}]$ plane; we have added the Ba II $\lambda 6496$ line and the $\mathrm{Eu}$ II $\lambda 6645$ line in this work (spectral synthesis was used to account for hyperfine/isotopic splitting). For metal-rich stars, Ba undergoes slow neutron capture; Eu is essentially a pure r-process neutron capture element.

To begin "tagging" the stars chemically, we first draw boundary lines by eye in the $[\mathrm{Ti} / \mathrm{Fe}]-[\mathrm{Fe} / \mathrm{H}]$ plane, separating the general regions for kicked-out disk, accreted, and in-situ halo stars. These lines are shown in the bottom panel of Figure 1. The orange, purple, and green filled circles in Figure 1 are stars taken from the literature for disk, halo, and satellite stars, respectively. In all three chemical planes shown in Figure 1, there is a fairly distinct region for satellite stars, although there is clearly some scattering of accreted stars into the kicked-out disk and in-situ regions.

If a star is tagged as a kicked-out disk or in-situ halo star in the $[\mathrm{Ti} / \mathrm{Fe}]-[\mathrm{Fe} / \mathrm{H}]$ plane, a more definitive classification can be made by seeing where it falls in the $[\mathrm{Ba} / \mathrm{Fe}]-[\mathrm{Fe} / \mathrm{H}]$ and $[\mathrm{Eu} / \mathrm{Fe}]-[\mathrm{Fe} / \mathrm{H}]$ planes. At these relatively high metallicities, both the $[\mathrm{Ba} / \mathrm{Fe}]-[\mathrm{Fe} / \mathrm{H}]$ and $[\mathrm{Eu} / \mathrm{Fe}]-[\mathrm{Fe} / \mathrm{H}]$ planes show a fairly distinct region for satellite stars, mainly from Fornax (Letarte et al. 2010) and the LMC (Pompéia et al. 2008). Using this technique, we first assess the stars classified as kicked-out disk stars in the $[\mathrm{Ti} / \mathrm{Fe}]-[\mathrm{Fe} / \mathrm{H}]$ plane. 

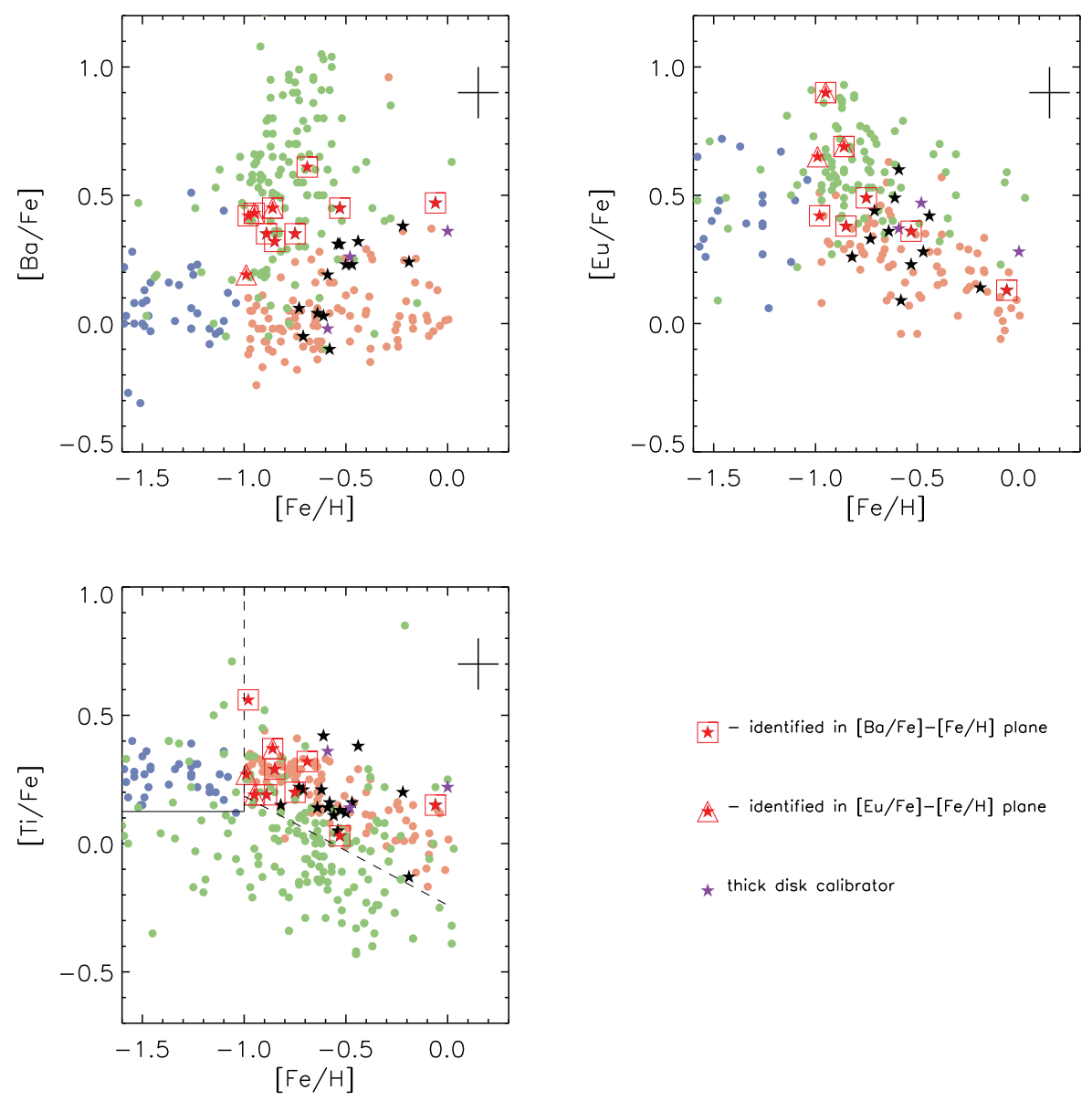

Figure 1. The $\alpha$-element and neutron capture chemical planes, with disk, halo, and accreted stars from the literature shown as the orange, blue, and green filled circles, respectively. The program stars falling into the kicked-out disk region in the titanium plane are shown as black or red filled stars. The red filled stars fall into the accreted region in either barium space (these are enclosed by a square) or europium space (these are enclosed by a triangle). The violet filled stars are thick disk calibrators.

These results are shown in Figure 1, where stars falling into the kicked-out disk region are shown as either black or red filled stars. The red stars fall into the accreted regions in either the $[\mathrm{Ba} / \mathrm{Fe}]-[\mathrm{Fe} / \mathrm{H}]$ or $[\mathrm{Eu} / \mathrm{Fe}]-[\mathrm{Fe} / \mathrm{H}]$ planes (the caption provides further details on the symbols). Of the 26 stars that fall into the kicked-out disk region based on their $[\mathrm{Ti} / \mathrm{Fe}]-[\mathrm{Fe} / \mathrm{H}]$ location, we reclassified 6 stars using this tagging technique as accreted rather than kicked-out disk stars.

The same technique was used to more accurately classify stars in the in-situ halo region, which can contain stars formed via any of the three scenarios (any kicked-out disk stars in this region would be due to a primordial merger). The results are summarized in Figure 2 , and we reclassified 8 of the 13 stars in this region as accreted, based on their location in the $[\mathrm{Ba} / \mathrm{Fe}]-[\mathrm{Fe} / \mathrm{H}]$ or $[\mathrm{Eu} / \mathrm{Fe}]-[\mathrm{Fe} / \mathrm{H}]$ planes.

\subsection{Assessing formation scenarios}

After adding the $[\mathrm{Ba} / \mathrm{Fe}]-[\mathrm{Fe} / \mathrm{H}]$ and $[\mathrm{Eu} / \mathrm{Fe}]-[\mathrm{Fe} / \mathrm{H}]$ planes, we reclassified 14 stars as accreted. Of the 66 stars analyzed, we find 40 (61\%) accreted, 21 (32\%) kicked-out disk, 

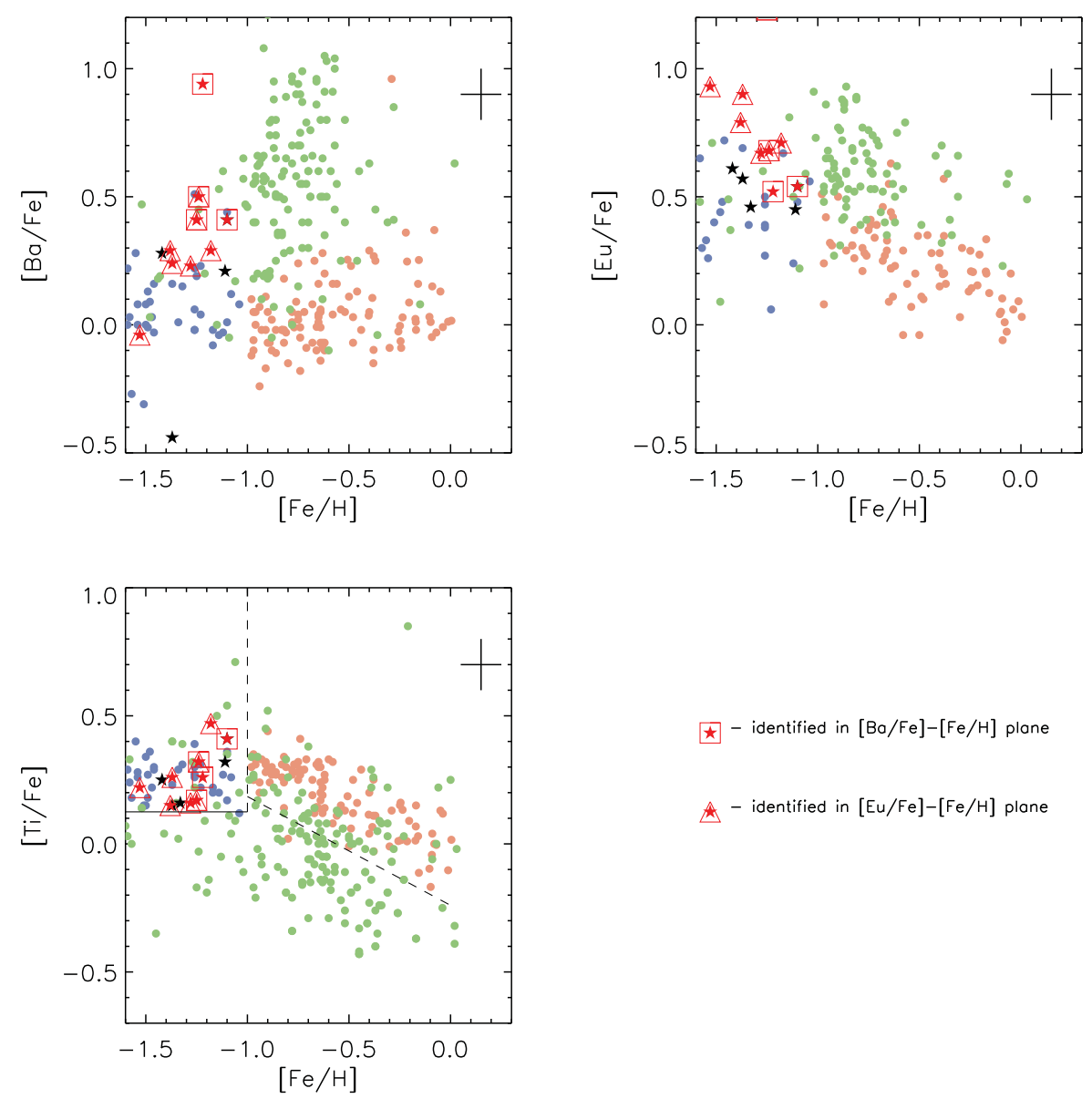

$\star-$ identified in $[\mathrm{Bo} / \mathrm{Fe}]-[\mathrm{Fe} / \mathrm{H}]$ plane
- identified in $[\mathrm{Eu} / \mathrm{Fe}]-[\mathrm{Fe} / \mathrm{H}]$ plane

Figure 2. Same as Fig. 1, but now highlighting stars falling into the in-situ halo region in the $[\mathrm{Ti} / \mathrm{Fe}]-[\mathrm{Fe} / \mathrm{H}]$ plane.

and $5(7 \%)$ in-situ halo. The results show a significant fraction of accreted stars in the inner halo (distances were found for the stars using the Dartmouth isochrones and are in the range of $\sim 1-10 \mathrm{kpc}$ ). This is similar to the results of Nissen \& Schuster's $(2010,2011)$ studies of dwarfs in the very nearby halo, where they also detect a significant presence of accreted stars.

To assess how many stars in the kicked-out disk region are actually kicked-out disk stars, as opposed to thick disk stars with high velocities, we defined a velocity $V^{\prime}$ to take into account the asymmetric drift: $V^{\prime}=v_{\text {hel }}+v_{\text {asymm }} \cos (b) \sin (l)$. The value of the dispersion that minimizes $\left|V^{\prime}\right|$ was found to be $\sigma=52.5 \mathrm{~km} / \mathrm{s}$ (Sheffield et al. 2012). In Figure 3, we show the distributions of $\left|V^{\prime}\right|$ for the entire medium-resolution sample and the 66 high-resolution stars for this study. The black dotted line is a Gaussian with a dispersion equal to $1.5 \sigma$, meant to represent the thick disk population. The kicked-out population is shown as the red distribution and, although we do find that roughly $10 \%$ are quite distinctly outside of the tail of the thick disk distribution, we still cannot claim conclusive evidence of a kicked-out disk population in the Milky Way without further statistical tests. 


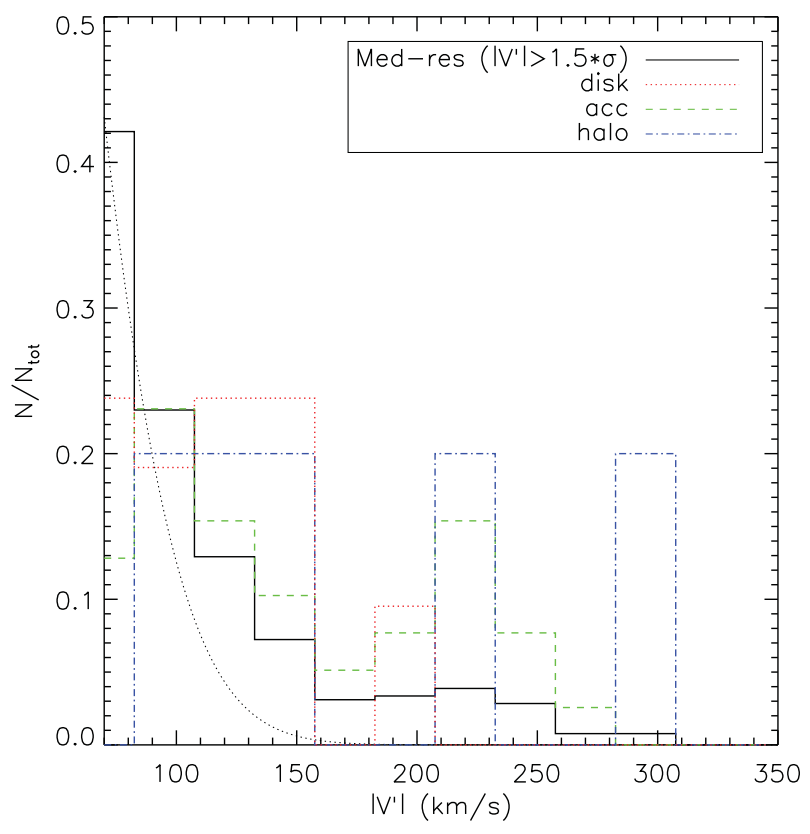

Figure 3. Distribution of the modulus of the asymmetric drift corrected radial velocities, $V^{\prime}$, for: all medium-resolution stars with $\left|V^{\prime}\right|>1.5-\sigma$ (solid black line), stars classified as kicked-out disk (red dotted line), stars classified as in-situ halo (blue dot-dashed line), and stars classified as accreted (green dashed line).

\section{Conclusions and Future Work}

Using chemical tagging, we have classified the formation scenarios for a sample of 66 $\mathrm{M}$ giants in the inner halo. We find that the majority of the stars (61\%) are classified as accreted. These results are consistent with the $\Lambda$ CDM paradigm whereby galaxies are built up hierarchically through mergers. Merger events dynamically impact the structure of a galaxy: for example, a merger can create "rings" of stars in the Galaxy (see, e.g., Xu et al. 2015 and Price-Whelan et al. 2015). Stars in these rings are thus a kicked-out disk population. Kinematically, a kicked-out population and an accreted population can be challenging to distinguish; the chemical abundance patterns of stars are thus invaluable in making this distinction. A small kicked-out population $(\sim 5 \%)$ has been detected in Andromeda (Dorman et al. 2013), a finding which is consistent with predictions from cosmological simulations (e.g., Tissera et al. 2013 find kicked-out disk populations of 1-30\% in their simulations of Milky Way-sized galaxies from the Aquarius Project). We find suggestive evidence for a small kicked-out disk population in the Milky Way.

The full high-resolution sample of $109 \mathrm{M}$ giants with the addition of more elements will be presented in an upcoming work.

\section{References}

Dorman, C. E., Widrow, L. M., Guhathakurta, P., et al. 2013, ApJ, 779, 103

Letarte, B., Hill, V., Tolstoy, E., et al. 2010, A\& A, 523, A17

Majewski, S. R., Skrutskie, M. F., Weinberg, M. D., \& Ostheimer, J. C. 2003, ApJ, 599, 1082

Nissen, P. E. \& Schuster, W. J. 2010, $A \mathscr{E} A, 511$, L10

Nissen, P. E. \& Schuster, W. J. 2011, $A \mathscr{E} A$, 530, A1

Pompéia, L., Hill, V., Spite, M., et al. 2008, A\&A, 480, 379 
Price-Whelan, A. M., Johnston, K. V., Sheffield, A. A., Laporte, C. F. P., \& Sesar, B. 2015, MNRAS, 452, 676

Read, J. I., Lake, G., Agertz, O., \& Debattista, V. P. 2008, MNRAS, 389, 1041

Sharma, S., Johnston, K. V., Majewski, S. R., Bullock, J., \& Muñoz, R. R. 2011, ApJ, 728, 106

Sheffield, A. A., Majewski, S. R., Johnston, K. V., et al. 2012, ApJ, 761, 161

Tissera, P. B., Scannapieco, C., Beers, T. C., \& Carollo, D. 2013, MNRAS, 432, 3391

Xu, Y., Newberg, H. J., Carlin, J. L., et al. 2015, ApJ, 801, 105 